\title{
Original
}

\section{Fast-track Surgery Protocol for Hepatectomy and the Rate of Surgical Site Infections: A Single-center Study}

\author{
Yuta EnAmi*, Takeshi Aoki, Tomokazu Kusano, \\ Koji Nogaki, Kazuhiro Matsuda, Kosuke YamadA, \\ Tomotake KoIzumi, Keitaro Mitamura, Akira Fujimori, \\ Reiko KoIKe, Satoru Goto, Makoto Watanabe, \\ Koji Otsuka and Masahiko Murakami
}

\begin{abstract}
The fast-track surgery protocol, including perioperative immunonutritional management, is increasingly gaining attention for the prevention of surgical site infections (SSIs). To analyze the association between the fast-track surgery protocol employed at a single center and outcomes, including SSIs and the length of hospital stays. This retrospective analysis included 217 patients who underwent hepatectomy at the study department between January 2009 and February 2014. Patients were divided into two groups: those managed by a conventional protocol (group $\mathrm{C}, \mathrm{n}=75$ ) and those managed by the fast-track surgery protocol (group $\mathrm{F}, \mathrm{n}=142)$. There were no significant differences in patient characteristics or factors between the two groups. However, serum albumin and total cholesterol levels before surgery were significantly higher in group $\mathrm{F}$ than in group $\mathrm{C}$, and pre-hepatectomy $\mathrm{C}$-reactive protein (CRP) levels were lower in group $\mathrm{F}$ than in group C. Moreover, serum albumin and CRP levels at postoperative day 7 were better in group $\mathrm{F}$ than in group $\mathrm{C}$. The operations were longer in group $\mathrm{F}$ than in group C (312 vs. $286 \mathrm{~min}$ ) and blood loss volume was less (385 g in group F vs. $428 \mathrm{~g}$ in group $\mathrm{C})$. SSI rates were significantly lower in group $\mathrm{F}(4.2 \%, \mathrm{n}=$ 6) than in group $\mathrm{C}(13.3 \%, \mathrm{n}=10)$, and the length of hospital stay was significantly shorter in group $\mathrm{F}$ (16.7 days) than in group $\mathrm{C}$ (25.8 days). The fast-track surgery protocol as a perioperative management strategy may improve preoperative nutritional status and postoperative inflammation, with subsequent reductions in SSI rates and the length of hospital stay in patients undergoing hepatectomy.
\end{abstract}

Key words : fast-track surgery, hepatectomy, surgical site infection, immunonutrition, albumin

\section{Introduction}

Hepatectomy is a highly invasive surgery compared with other gastroenterological surgeries; the incision tends to be larger during open hepatectomy, bringing an increased risk of several

Department of Gastrointestinal and General Surgery, Showa University School of Medicine, 1-5-8 Hatanodai, Shinagawa-ku, Tokyo 142-8666, Japan.

* To whom corresponding should be addressed. 
complications. Surgical site infection (SSI) is one of the most frequent complications of hepatectomy ${ }^{1-3)}$. SSIs can be fatal and are associated with prolonged hospital stays and increased medical expenses. Recently, the "fast-track surgery" protocol, which includes perioperative immunonutritional management, has gained attention because of its focus on the prevention of SSIs ${ }^{4-6)}$. At our department, the fast-track surgery protocol was introduced in 2011 for patients' early mobilization and discharge ${ }^{5)}$. We herein report our retrospective analysis of the validity of fast-track surgery and its association with SSIs in our department.

\section{Materials and methods}

The clinical data of 217 patients who underwent hepatectomy at Showa University Hospital between January 2009 and February 2014 were retrospectively analyzed. Patients who underwent emergency surgery or surgery for trauma were excluded. Patients were categorized into group C, all 75 consecutive patients who underwent conventional perioperative management in 2009 and 2010, and group F, all 142 consecutive patients who underwent the fast-track surgery protocol after its implementation in 2011.

The perioperative management, for the conventional protocol and the fast-track surgery protocol are presented in Table 1. A guidebook on liver disease, hepatectomy, and the perioperative course was provided to patients while attending the outpatient clinic. Patients were placed on $\mathrm{ANOM}^{\circledR}$ oral nutrition (Otsuka Pharmaceutical Factory, Naruto, Japan) and initiated on walking and breathing training 7 days before surgery. The day before surgery, the patients took a shower, but the hair on the surgical site was not shaved. No more than $30 \mathrm{~min}$ before the skin incision, patients were administered $1 \mathrm{~g}$ cefmetazole as a surgical antimicrobial prophylaxis, and additional doses were administered every $3 \mathrm{~h}$ during surgery. After the surgical incision was closed, the subcutaneous tissue was washed with warm saline solution. Skin closure was achieved with subcuticular sutures using 4-0 PDS $^{\circledR}$ (Ethicon) and DuoACTIVE ${ }^{\circledR}$ ET (ConvaTec, Flintshire, UK) was used as a sterile dressing for $48 \mathrm{~h}$ after the primary closure of skin incision. In surgeries with laparotomy incisions, AQUACEL ${ }^{\circledR} \mathrm{Ag}$ (ConvaTec) was also used. Postoperatively, patients resumed breathing training using $\mathrm{COACH} 2^{\circledR}$ (Smiths Medical, OH, USA) and walking training; the goal for the third day after the operation was 35 laps of the ward $(80 \mathrm{~m}$ per lap). All patients started walking and breathing training on postoperative day 1 and peroral feeding on the morning of postoperative day 1 . There was no significant difference between the management of groups $\mathrm{C}$ and $\mathrm{F}$, except for the above protocols.

SSIs were defined by following the Centers for Diseases Control and Prevention guidelines ${ }^{7)}$. Purulent drainage from superficial and deep incisions indicated superficial and deep incisional SSIs, respectively. Moreover, purulent drainage from a drain placed through an incision into the organ or space indicated organ/space incisional SSI.

All values are presented as mean \pm standard deviation. Data were analyzed using the $\mathrm{JMP}^{\mathbb{R}}$ software (version 10.0.0 for Mac; SAS Institute, Cary, NC, USA). Individual parameters in the two groups were compared using Student's t test, Welch's t test, or Pearson's chi-square test. $p$ $<0.05$ was considered statistically significant. 
Table 1

Our fast-track protocol

Days before surgery A guidebook on liver disease, hepatectomy, and the perioperative course was provided to patients while attending the outpatient clinic.

Patients took $\mathrm{ANOM}^{\circledR}$ for 7 days before their operation.

Patients began breathing training by $\mathrm{COACH} 2{ }^{\circledR} 7$ days before their operation.

Patients began walking training 7 days before operation.

One day before surgery Patients took a shower, but the hair on the surgical site was not shaved.

The day of surgery No more than $30 \mathrm{~min}$ before the skin incision, patients were administered $1 \mathrm{~g}$ cefmetazole as surgical antimicrobial prophylaxis, and additional doses were administered every $3 \mathrm{~h}$ during surgery.

After the surgical incision was closed, the subcutaneous tissue was washed with warm saline solution.

Skin closure was achieved with subcuticular sutures using 4-0 $\operatorname{PDS}^{\circledR}$ (Ethicon).

DuoACTIVE $^{\circledR}$ ET (ConvaTec, UK) was used as a sterile dressing.

In surgeries with laparotomy incisions, AQUACEL ${ }^{\circledR} \mathrm{Ag}$ (ConvaTec) was added.

Days after surgery All patients started walking and breathing training on postoperative day 1 and peroral feeding on the morning of postoperative day 1 .

Conventional protocol

Days before surgery

Informed consent about the operation and complications was obtained from patients and their family.

One day before surgery Patients took a shower if they needed to. The hair on the surgical site was shaved.

The day of surgery No more than 30 min before the skin incision, patients were administered $1 \mathrm{~g}$ flomoxef sodium as a surgical antimicrobial prophylaxis.

After the surgical incision was closed, the subcutaneous tissue was washed with warm saline solution.

Days after surgery Patients started walking and breathing training on postoperative day 3 or 4 and peroral feeding on the morning of postoperative day 2 .

The Committee on Ethics of Showa University Medical School and Institutional Review Board reviewed and approved the study protocols (approval code: No. 3047).

\section{Results}

There were no significant differences between group $\mathrm{C}$ and group $\mathrm{F}$ in patient characteristics or tumor-related factors (Tables 2, 3, 4-1 and 4-2). Analysis of the preoperative data revealed that the mean serum albumin and mean total cholesterol levels were significantly higher in group F than in group C (3.97 vs. $3.71 \mathrm{~g} / \mathrm{dL}$ and 184 vs. $156 \mathrm{mg} / \mathrm{dL}$, respectively) (Table 2). The mean C-reactive protein (CRP) of group F $(0.58 \mathrm{mg} / \mathrm{dL})$ was lower than that of group C $(1.23 \mathrm{mg} /$ $\mathrm{dL}$ ), although this difference was not statistically significant. Furthermore, on postoperative day 7, the mean serum albumin level was higher, and the mean CRP level was lower in group F than in group C, albeit without statistical significance (Table 2).

Next, we analyzed data of only those patients undergoing open hepatectomy (54 patients in 
Table 2

\begin{tabular}{llll} 
Characteristics of patients & Group C $(\mathrm{n}=75)$ & Group F $(\mathrm{n}=142)$ & $p$ value \\
\hline Sex (male/female), $\mathrm{n}$ & $46 / 29$ & $91 / 51$ & 0.6912 \\
Age (years) & $67.5 \pm 11.0$ & $68.8 \pm 9.9$ & 0.3790 \\
Disease (HCC/meta/others), $\mathrm{n}$ & $34 / 29 / 12$ & $57 / 60 / 25$ & 0.8466 \\
Number of tumors, n & $1.76 \pm 1.45$ & $1.61 \pm 1.30$ & 0.4432 \\
Maximum diameter of tumor (mm) & $44.4 \pm 29.6$ & $44.4 \pm 31.3$ & 0.9893 \\
ICG15R (\%) & $13.5 \pm 8.2$ & $12.6 \pm 8.5$ & 0.4775 \\
Child-Pugh (A/B), n & $69 / 6$ & $133 / 9$ & 0.6462 \\
\hline
\end{tabular}

\begin{tabular}{llll} 
Laboratory data before operation & Group C $(\mathrm{n}=75)$ & Group F $(\mathrm{n}=142)$ & $p$ value \\
\hline Albumin $(\mathrm{g} / \mathrm{dL})$ & $3.71 \pm 0.52$ & $3.97 \pm 0.53$ & $0.0005^{*}$ \\
CRP $(\mathrm{mg} / \mathrm{dL})$ & $1.23 \pm 2.75$ & $0.58 \pm 1.98$ & 0.0967 \\
Cholesterol $(\mathrm{mg} / \mathrm{mL})$ & $156 \pm 42.7$ & $184 \pm 35.0$ & $0.0002^{*}$ \\
\hline
\end{tabular}

\begin{tabular}{llll} 
Laboratory data 7 days after operation & Group C $(\mathrm{n}=75)$ & Group F $(\mathrm{n}=142)$ & $p$ value \\
\hline Albumin $(\mathrm{g} / \mathrm{dL})$ & $3.15 \pm 0.44$ & $3.25 \pm 0.47$ & 0.1459 \\
CRP $(\mathrm{mg} / \mathrm{dL})$ & $3.97 \pm 4.18$ & $3.21 \pm 3.74$ & 0.1776 \\
\hline
\end{tabular}

\begin{tabular}{llll} 
Operative variables & Group C $(\mathrm{n}=75)$ & Group F $(\mathrm{n}=142)$ & $p$ value \\
\hline Operative time $(\mathrm{min})$ & $286 \pm 123$ & $312 \pm 189$ & 0.2509 \\
Operative blood loss $(\mathrm{g})$ & $428 \pm 510$ & $385 \pm 624$ & 0.6236 \\
Rate of laparoscopic surgery (\%) & $28.0 \%(\mathrm{n}=21)$ & $46.5 \%(\mathrm{n}=66)$ & $0.0065^{*}$ \\
\hline
\end{tabular}

\begin{tabular}{llll} 
Patient outcomes & Group C $(\mathrm{n}=75)$ & Group F $(\mathrm{n}=142)$ & $p$ value \\
\hline Rate of SSI $(\%)$ & $13.3 \%(\mathrm{n}=10)$ & $4.2 \%(\mathrm{n}=6)$ & $0.0366^{*}$ \\
Hospital stay (day) & $25.8 \pm 25.2$ & $16.7 \pm 11.0$ & $0.0039^{*}$ \\
\hline & & Mean \pm SD & $* p<0.05$
\end{tabular}

Group $\mathrm{C}=$ Conventional protocol

Group $\mathrm{F}=$ Fast-track protocol

group $\mathrm{C}$ and 76 patients in group $\mathrm{F}$ ). These findings are presented in Table 3. There were no significant differences in the patient characteristics or tumor-related factors. The mean preoperative serum albumin and total cholesterol levels were significantly higher in group $\mathrm{F}$ than in group C. Albeit not statistically significant, the mean CRP level in group F was lower than that in group C. On postoperative day 7, the mean albumin and CRP levels in group F were higher than those in group $\mathrm{C}$, although these differences were not statistically significant.

We further analyzed data of only those patients undergoing laparoscopic liver resection (21 patients in group $\mathrm{C}$ and 66 patients in group F). These findings are presented in Table 4-1. We did not find any significant differences in patient characteristics between the groups except 
Table 3

\begin{tabular}{llll} 
Characteristics of patients (open surgery) & Group C $(\mathrm{n}=54)$ & Group F $(\mathrm{n}=76)$ & $p$ value \\
\hline Sex (male/female), $\mathrm{n}$ & $35 / 19$ & $46 / 30$ & 0.6223 \\
Age (years) & $68.0 \pm 11.6$ & $69.4 \pm 8.2$ & 0.4372 \\
Disease (HCC/meta/others), $\mathrm{n}$ & $21 / 22 / 11$ & $24 / 34 / 18$ & 0.6839 \\
Number of tumors, n & $1.94 \pm 1.68$ & $1.96 \pm 1.64$ & 0.5165 \\
Maximum diameter of tumor (mm) & $51.3 \pm 32.1$ & $49.3 \pm 34.3$ & 0.7612 \\
ICG15R (\%) & $13.5 \pm 8.9$ & $11.9 \pm 6.9$ & 0.2569 \\
Child-Pugh (A/B), n & $49 / 5$ & $71 / 5$ & 0.5720
\end{tabular}

\begin{tabular}{llll} 
Laboratory data before operation (open surgery) & Group C $(\mathrm{n}=54)$ & Group F $(\mathrm{n}=76)$ & $p$ value \\
\hline Albumin $(\mathrm{g} / \mathrm{dL})$ & $3.68 \pm 0.54$ & $3.94 \pm 0.53$ & $0.0075^{*}$ \\
CRP (mg/dL) & $1.63 \pm 3.22$ & $0.58 \pm 2.42$ & 0.0704 \\
Cholesterol (mg/mL) & $161 \pm 39.3$ & $192 \pm 37.5$ & $0.0024^{*}$
\end{tabular}

\begin{tabular}{llll} 
Laboratory data 7 days after operation (open surgery) & Group C $(\mathrm{n}=54)$ & Group F $(\mathrm{n}=76)$ & $p$ value \\
\hline Albumin $(\mathrm{g} / \mathrm{dL})$ & $3.11 \pm 0.40$ & $3.15 \pm 0.47$ & 0.6251 \\
CRP $(\mathrm{mg} / \mathrm{dL})$ & $4.21 \pm 4.39$ & $3.88 \pm 4.43$ & 0.6770 \\
\hline
\end{tabular}

\begin{tabular}{llll} 
Operative variables (open surgery) & Group C $(\mathrm{n}=54)$ & Group F $(\mathrm{n}=76)$ & $p$ value \\
\hline Operative time $(\mathrm{min})$ & $319 \pm 121$ & $394 \pm 191$ & $0.0095^{*}$ \\
Operative blood loss $(\mathrm{g})$ & $510 \pm 559$ & $485 \pm 604$ & 0.8128 \\
\hline
\end{tabular}

\begin{tabular}{|c|c|c|c|}
\hline Patient outcomes (open surgery) & Group C $(\mathrm{n}=54)$ & Group F $(\mathrm{n}=76)$ & $p$ value \\
\hline Rate of SSI (\%) & $18.5 \% \quad(\mathrm{n}=10)$ & $6.6 \%(\mathrm{n}=5)$ & 0.0520 \\
\hline Hospital stay (day) & $28.9 \pm 27.2$ & $19.4 \pm 11.9$ & $0.0193^{*}$ \\
\hline & & Mean \pm SD & $* ; p<0.05$ \\
\hline & \multicolumn{3}{|c|}{$\begin{array}{l}\text { Group } \mathrm{C}=\text { Conventional protocol } \\
\text { Group } \mathrm{F}=\text { Fast-track protocol }\end{array}$} \\
\hline
\end{tabular}

the mean tumor diameter, which was larger in group $\mathrm{F}$ than in group $\mathrm{C}$. Of the preoperative laboratory parameters, the mean total cholesterol level was significantly higher in group $\mathrm{F}$ than in group C. Additionally, the mean preoperative serum albumin level was higher and the CRP level was lower in group $\mathrm{F}$ than in group $\mathrm{C}$. Although not statistically significant, the mean serum albumin and CRP levels on postoperative day 7 were better in group $\mathrm{F}$ than in group $\mathrm{C}$.

Further analysis of the operative factors in all patients revealed that the operation time was longer and blood loss was less in group $\mathrm{F}$ than in group $\mathrm{C}$, although this was not statistically significant (312 vs. $286 \mathrm{~min}$ and 385 vs. $428 \mathrm{~g}$, respectively) (Table 2). Similarly, among the patients who underwent open surgery, the operation time was longer (394 vs. 319 min) and the blood loss was less (485 vs. $510 \mathrm{~g}$ ) in group $\mathrm{F}$ than in group C. Finally, analysis of patients 
Table 4-1

Characteristics of patients (laparoscopic surgery)

Sex (male/female), $\mathrm{n}$

Group C $(\mathrm{n}=21)$

Age (years)

$11 / 10$

$66.1 \pm 9.6$

$45 / 21$

$p$ value

Disease (HCC/meta/others), $n$

$12 / 7 / 2$

$68.1 \pm 11.5$

0.1921

Number of tumors, $\mathrm{n}$

$1.33 \pm 0.48$

$33 / 26 / 7$

0.4775

Maximum diameter of tumor (mm)

$27.7 \pm 10.9$

$1.19 \pm 0.46$

0.8481

ICG15R (\%)

$13.3 \pm 6.3$

$38.7 \pm 26.6$

0.2659

Child-Pugh (A/B), n

20/1

$13.6 \pm 10.0$

$0.0160^{*}$

0.9699

$62 / 4$

0.8238

Laboratory data before operation (laparoscopic surgery)

Group C $(\mathrm{n}=21)$

Group F $(\mathrm{n}=66)$

$p$ value

Albumin (g/dL)

$3.79 \pm 0.48$

$4.02 \pm 0.52$

0.0790

CRP (mg/dL)

$0.40 \pm 1.04$

$0.57 \pm 1.32$

0.5937

Cholesterol $(\mathrm{mg} / \mathrm{mL})$

$147 \pm 49.5$

$178 \pm 32.1$

$0.0446^{*}$

Laboratory data 7 days after operation (laparoscopic surgery)

Albumin (g/dL)

Group C $(\mathrm{n}=21)$

Group F $(\mathrm{n}=66)$

$p$ value

CRP $(\mathrm{mg} / \mathrm{dL})$

$3.26 \pm 0.53$

$3.36 \pm 0.44$

0.3688

$3.35 \pm 3.62$

$2.44 \pm 2.56$

0.2938

\begin{tabular}{llll} 
Operative variables (laparoscopic surgery) & Group C $(\mathrm{n}=21)$ & Group F $(\mathrm{n}=66)$ & $p$ value \\
\hline Operative time (min) & $205 \pm 85.8$ & $218 \pm 137$ & 0.5987 \\
Operative blood loss $(\mathrm{g})$ & $223 \pm 279$ & $264 \pm 631$ & 0.6907 \\
\hline
\end{tabular}

\begin{tabular}{llll} 
Patient outcomes (laparoscopic surgery) & Group C $(\mathrm{n}=21)$ & Group F $(\mathrm{n}=66)$ & $p$ value \\
\hline Rate of SSI (\%) & $0 \%(\mathrm{n}=0)$ & $1.5 \%(\mathrm{n}=1)$ & 0.5757 \\
Hospital stay (day) & $17.8 \pm 17.6$ & $13.7 \pm 9.2$ & 0.3241 \\
\hline & & Mean \pm SD & $* p<0.05$
\end{tabular}

Group $\mathrm{C}=$ Conventional protocol

Group $\mathrm{F}=$ Fast-track protocol

Table 4-2

Outcomes of patients (Group F)

Open surgery

$(\mathrm{n}=76)$
Laparoscopic surgery

$(\mathrm{n}=66)$

Rate of SSI (\%)

$6.6 \%(\mathrm{n}=5)$

$1.5 \%(\mathrm{n}=1)$

0.1701 
Table 5

Multivariate analysis of SSI risk factors in all patients

\begin{tabular}{llll}
\hline Variables & Odds ratio & $95 \%$ Confidence intervals & $p$ value \\
\hline Perioperative albumin value & 0.071276 & $0.006459-0.786486$ & $0.0244^{*}$ \\
Perioperative cholesterol value & 12.80627 & $0.998147-1.020609$ & 0.0863 \\
\hline & & & $* ; p<0.05$
\end{tabular}

undergoing laparoscopic surgery also revealed that there was a tendency for longer operation times but in this case more blood loss in group F than in group C (218 vs. $205 \mathrm{~min}$ and 264 vs. $223 \mathrm{~g}$, respectively) (Table 4-1). Considering all patients, the rates of laparoscopic surgery were significantly higher in group $\mathrm{F}$ than group $\mathrm{C}(46.5 \%$ and $28.0 \%$, respectively) (Table 2$)$.

SSI rates were significantly lower in group $\mathrm{F}$ than group $\mathrm{C}$, being $4.2 \%(\mathrm{n}=6)$ and $13.3 \%$ $(\mathrm{n}=10)$, respectively (Table 2$)$. All patients with SSIs in group $\mathrm{C}$ and five of the six patients with SSIs in group $\mathrm{F}$ had undergone open surgery. Limiting the analysis of SSIs to the subset of patients undergoing open surgery revealed that there were fewer SSIs in group $\mathrm{F}$ than in group C $(6.6 \%(\mathrm{n}=5)$ vs. $18.5 \%(\mathrm{n}=10), p=0.052) \quad$ (Table 3). Finally, only one patient undergoing laparoscopic hepatectomy in group $\mathrm{F}$ had an SSI, and no patients undergoing laparoscopic hepatectomy in group C developed SSIs (Table 4-1). There was no significant difference between open surgery and laparoscopic surgery in the rate of SSI in group F (Table 4-2).

The length of hospital stay was significantly shorter in group F than in group C (16.7 vs. 25.8 days, respectively) (Table 2). This finding held true in the patients undergoing open surgery (group F 19.4 vs. group C 28.9 days; $p=0.0193$ ) (Table 3). Among the patients undergoing laparoscopic surgery, the length of hospital stay, albeit shorter in group $\mathrm{F}$ than in group $\mathrm{C}$, did not significantly differ between the two groups (Table 4-1).

Evaluation of factors associated with SSIs by multivariate analysis revealed that preoperative serum albumin was the only risk factor for SSIs in the study cohort (Table 5).

\section{Discussion}

The aim of the present study was to determine whether the fast-track surgery protocol employed at our department with perioperative management including immunonutrition prevented SSIs after hepatectomy. Our analysis revealed that SSI rates were significantly lower and the length of hospital stay was significantly shorter in patients who were managed by the fast-track surgery protocol. Previous studies have reported several factors, including perioperative nutritional status, liver function, operation time, blood loss, repeat hepatectomy, and bile fistula, as risk factors for SSIs after hepatectomy ${ }^{1-3,8)}$. In the present study, preoperative serum albumin and total cholesterol levels were significantly better in the fast-track group than in the conventional group, suggesting the possibility that the administration of immunonutrition for preoperative patients in the fast-track group causes the elevation of these values. Serum albumin and total cholesterol levels reflect nutritional status and might have contributed to the prevention of SSIs. The study results indicate that the preoperative management including oral immuno- 
nutrition employed at our department was successful in preventing SSIs. Several meta-analyses have previously indicated that preoperative immunonutrition is an excellent approach to prevent infectious complications after elective surgery and to shorten the length of hospital stays ${ }^{9,10)}$, but despite abundant information regarding its utility in gastrointestinal surgery, data on its efficacy in liver surgery are limited. In a clinical study on liver transplantation surgery, immunonutrition was shown to reduce postoperative infectious complications ${ }^{11)}$, and several studies suggest its efficacy in preoperative venous or oral nutritional management ${ }^{12-17)}$. The ESPEN guidelines recommend immunonutritional therapy for major surgery with a high risk of infection, regardless of nutritional risk, and recommend starting this therapy 5-7 days before elective surgery ${ }^{18)}$. In previous reports, the administration of $220-1,000 \mathrm{~mL} /$ day of immune-enhancing diet (IED) from 5 days to 3 weeks before surgery reduced CRP after hepatectomy ${ }^{17)}$ and risk of infection after gastric $^{19)}$ and colorectal ${ }^{20)}$ operations. It is reported that such diets are useful for reducing infectious diseases. We therefore hypothesized that we could reduce the risk of developing infectious complications after hepatectomy by administering $200-600 \mathrm{~mL} /$ day of $\mathrm{ANOM}^{\circledR}$ as an IED from 7 days before surgery.

In the present study, we implemented perioperative management including oral immunonutrition before hepatectomy. The significantly better preoperative serum albumin and total cholesterol levels we found as indices of nutritional status ${ }^{21}$ might be associated with the lower SSI rates and shorter hospital stays in patients managed by the fast-track surgery protocol. Moreover, improvements in preoperative inflammatory changes may contribute to the improvement of nutritional status. High preoperative CRP levels and hypoalbuminemia were previously reported to be poor prognostic factors in hepatectomy ${ }^{22}$. Therefore, perioperative management including oral immunonutrition should be considered as a useful approach to control high CRP levels and hypoalbuminemia.

In the present study, patients were started on $\mathrm{ANOM}^{\circledR} 7$ days before surgery as an addition to their normal diet. $\mathrm{ANOM}^{\circledR}$ contains $20 \%$ protein and $25 \%$ lipids, wherein percentages reflect the energy density. And it contains glutamine and arginine as amino acids, and the lipids are $\omega-3$ and $\omega-6$ fatty acids (with a ratio of $1: 2$ ). The primary $\omega-3$ fatty acid in ANOM $^{\mathbb{R}}$ is $\alpha$ -linolenic acid, which is present in $\mathrm{ANOM}^{\circledR}$ at a higher percentage than either eicosapentaenoic acid (EPA) or docosahexaenoic acid (DHA) (Table 6). $\alpha$-Linolenic acid is the precursor of EPA and DHA, which have anti-inflammatory functions by suppressing the arachidonic cascade, i.e., the production and subsequent effect of inflammatory eicosanoids. One study reported that parenteral nutrition supplemented with the emulsion of fish oil, which includes EPA and DHA rich in $\omega-3$ fatty acids, reduced systemic inflammatory response syndrome and the length of hospital stays in patients with gastric and colonic cancer ${ }^{23)}$. In the present study, the improvement in immunocompetence and observed anti-inflammatory effects reflected by higher serum albumin and lower CRP levels might be due to the oral intake, and several reports indicated that preoperative nutritional condition could be improved by the oral administration of immunonutrition. Moreover, in the present study, our perioperative management was associated with reduced SSI rates and shorter length of hospital stays, and perioperative serum albumin level was significantly 
Table 6

\begin{tabular}{|c|c|c|}
\hline Component composition of ANOM & Amount per $100 \mathrm{~g}$ & \\
\hline Energy（kcal） & 100 & $100 \%$ \\
\hline Protein $(\mathrm{g})$ & 5 & $20 \%$ \\
\hline Glutamine (g) & 0.75 & \\
\hline Arginine (g) & 0.46 & \\
\hline Fat $(\mathrm{g})$ & 2.8 & $25 \%$ \\
\hline$\omega-6$ Fatty acid family (g) & 0.29 & $2.61 \%$ \\
\hline (Linoleic acid) & & $2.61 \%$ \\
\hline$\omega-3$ Fatty acid family $(\mathrm{g})$ & 0.15 & $1.35 \%$ \\
\hline$(\alpha$-Linolenic acid $)$ & & $1.04 \%$ \\
\hline (Eicosapentaenoic acid) & & $0.18 \%$ \\
\hline (Docosahexaenoic acid) & & $0.12 \%$ \\
\hline$\omega-6: \omega-3$ & $2: 1$ & \\
\hline
\end{tabular}

associated with the reduced SSI rates after hepatectomy by the multivariate analysis. Future studies are warranted to reveal the specific nutritional components associated with this outcome.

In the study cohort, the rate of laparoscopic hepatectomy was significantly higher in the fast-track group than in the conventional group, which might be the main contributor to the improved SSI rate found in the fast-track group. A previous study has suggested that laparoscopic hepatectomy might be a factor of reducing SSI ${ }^{24)}$, but the length of hospital stays, preoperative serum albumin, and total cholesterol levels did not significantly differ between patients using the fast-track or conventional protocol within the subset undergoing laparoscopic surgery. One potential reason for this outcome is that almost all patients undergoing laparoscopic surgery were in a moderately good clinical condition preoperatively during the time period when the conventional method was used for their management. Conversely, the rate of SSI in the open surgery subset in the conventional protocol may be acceptable based on previous reports showing that the rate of SSI in open hepatectomy was $24.8 \%(29 / 117)^{24)}$ and the complication rate was $27 \%(129 / 474)^{25)}$. In the present study, in the subset of patients undergoing open surgery, the hospital stay was significantly shorter and the SSI rate was lower $(p=0.052)$ in the fasttrack group than the conventional group, which might reflect a significantly better preoperative nutritional status in these patients, although the operation time was longer in patients undergoing open surgery with the fast-track protocol. Overall, improvement of the preoperative nutritional status by oral administration might have contributed to the reduced SSI rate despite the longer operation time, particularly among patients undergoing open surgery.

Postoperative fast-track program and enhanced recovery after surgery are recently proposed perioperative management approaches that have been increasingly used worldwide ${ }^{4-6)}$. Initially used primarily in patients undergoing colon surgery, these protocols have also been successfully used in gastric and breast surgery ${ }^{26-28)}$. Although there have been only a small number of studies on liver surgery, these protocols have been found to be feasible and effective after liver 
resection $^{25)}$. In the present study, all patients were provided information regarding the surgery and protocols before surgery, and walking and breathing training was started 7 days before surgery. Moreover, patients were started on oral nutrition as well as walking and breathing training on postoperative day 1 . We had induced and performed the fast-track protocol, including management of the closing incision. Therefore, several factors might have contributed to the prevention of SSIs. We can conclude that the implementation of the fast-track surgery protocol at our department was safe, with no mortality, and might have greatly contributed to the shortening of the postoperative hospitalization period and reduction of SSI rates. However, this was a retrospective study including patients with different etiologies ranging from metastatic liver tumor to hepatocellular carcinoma. Whether the fast-track surgery protocol implemented at our department can be introduced to all patients, including those with poor liver function, requires further studies with larger patient cohorts.

The limitations of our study are that the analysis was limited to a single institution and that the study had a retrospective design. Further analysis and more patients are needed to confirm the effect of our protocol.

\section{Conclusion}

The fast-track surgery protocol including perioperative management with walking and breathing training and preoperative nutrition is a feasible and safe approach that can improve preoperative nutritional status, particularly preoperative albumin level, and postoperative inflammation, thereby contributing to reduced SSI rates and shorter hospital stays in patients undergoing hepatectomy.

\section{Conflict of interest}

The authors declare no conflict of interest.

Informed consent was obtained from all individual participants included in this study.

\section{References}

1) Uchiyama K, Ueno M, Ozawa S, et al. Risk factors for postoperative infectious complications after hepatectomy. $J$ Hepatobiliary Pancreat Sci. 2011;18:67-73.

2) Akikawa T, Kurokawa T, Ohwa Y, et al. Risk factors for surgical site infection after hepatectomy for hepatocellular carcinoma. Hepatogastroenterology. 2011;58:143-146.

3) Sadamori H, Yagi T, Shinoura S, et al. Risk factors for major morbidity after liver resection for hepatocellular carcinoma. Br J Surg. 2013;100:122-129.

4) Kehlet H. Multimodal approach to control postoperative pathophysiology and rehabilitation. Br J Anaesth. 1997;78:606-617.

5) Wilmore DW, Kehlet H. Management of patients in fast track surgery. BMJ. 2001;322:473-476.

6) Fearon KC, Ljungqvist O, Von Meyenfeldt M, et al. Enhanced recovery after surgery: a consensus review of clinical care for patients undergoing colonic resection. Clin Nutr. 2005;24:466-477.

7) Mangram AJ, Horan TC, Pearson ML, et al. Guideline for prevention of surgical site infection, 1999. Hospital Infection Control Practices Advisory Committee. Infect Control Hosp Epidermiol. 1999;20:250-278;quiz279-280. 
8) Sakamoto K, Tamesa T, Maeda Y, et al. Risk factors for surgical site infection after hepatectomy. J Jpn Soc Surg Infect. 2013;10:251-257.

9) Heys SD, Walker LG, Smith I, et al. Enteral nutritional supplementation with key nutrients in patients with critical illness and cancer: a meta-analysis of randomized controlled clinical trials. Ann Surg. 1999;229:467-477.

10) Heyland DK, Novak F, Drover JW, et al. Should immunonutrition become routine in critically ill patients? A systematic review of the evidence. JAMA. 2001;286:944-953.

11) Plank LD, McCall JL, Gane EJ, et al. Pre- and postoperative immunonutrition in patients undergoing transplantation: a pilot study of safety and efficancy. Clin Nutr. 2005;24:288-296.

12) Fan ST, Lo CM, Lai EC, et al. Perioperative nutritional support in patients undergoing hepatectomy for hepatocellular carcinoma. N Engl J Med. 1994;331:1547-1552.

13) Fukushima R, Iwasaki K, Inaba T. Clinical effect of immunonutrition, Japanese evidence. J Jpn Soc Parenter Enteral Nutr. 2007;22:283-288. (in Japanase).

14) Haji S, Ohyanagi H. Significance of immunonutrition on attenuation of surgical infection after liver resection from the viewpoint of immunological and metabolic preconditioning. J Jpn Soc Surg Infect. 2010;7:231-237.

15) Haji S, Ohyanagi H. Perioperative enteral administration of ohm 3 fatty acids in immunonutrition for patients undergoing hepatectomy. Jpn J Surg Metab Nutr. 2010;44:111-118.

16) Haji S. Kansetsujo ni okeru Immunonutrition. Kan Tan Sui. 2011;63:417-423. (in Japanase).

17) Ishizaki M, Kaibori M, Matsui K, et al. Shinkou-kansaibougan no shujutsuki narabini jutsugo-kagakuryoho-shikouji ni okeru gan-men'eki-eiyou-ryoho ni kansuru kento. The Japanese Journal of Clinical Nutrition. 2014;125:163-168. (in Japanase).

18) Weimann A, Braga M, Harsanyi L, et al. ESPEN guidelines on Enteral nutrition: surgery including organ transplantation. Clin Nutr. 2006;25:224-244.

19) Gianotti L, Braga M, Nespoli L, et al. A randomized controlled trial of preoperative oral supplementation with a specialized diet in patients with gastrointestinal cancer. Gastroenterology. 2002;122:1763-1770.

20) Braga M, Gianotti L, Vignali A, et al. Preoperative oral arginine and n-3 fatty acid supplementation improves the immunometabolic host response and outcome after colorectal resection for cancer. Surgery. 2002;132:805-814.

21) Katsuramaki T, Hirata K, Ura H. Kanzo shujutsu. Jpn J Nutr Assess. 2001;18:391-397. (in Japanase).

22) Ishizuka M, Kubota K, Kita J, et al. Usefulness of a modified inflammation-based prognostic system for predicting postoperative mortality of patients undergoing surgery for primary hepatocellular carcinoma. J Surg Oncol. 2011;103:801-806.

23) Jiang ZM, Wilmore DW, Wang XR, et al. Randomized clinical trial of intravenous soybean oil alone versus soybean oil plus fish oil emulsion after gastrointestinal cancer surgery. Br J Surg. 2010;97:804-809.

24) Kanazawa A, Sakae M, Tsukamoto T, et al. Surgical site infection after laparoscopic liver resection. J Jpn Soc Surg Infect. 2014;11:311-317.

25) Wu SJ, Xiong XZ, Lu J, et al. Fast-track programs for liver surgery: a meta-analysis. J Gastrointest Surg. 2015;19:1640-1652.

26) Varadhan KK, Neal KR, Dejong $\mathrm{CH}$, et al. The enhanced recovery after surgery (ERAS) pathway for patients undergoing major elective open colorectal surgery; a meta-analysis of randomized controlled trials. Clin Nutr. 2010;29:434-440.

27) Arsalani-Zadeh R, EIFadl D, Yassin N, et al. Evidence-based review of enhancing postoperative recovery after breast surgery. Br J Surg. 2011;98:181-196.

28) Yamada $\mathrm{T}$, Hayashi $\mathrm{T}$, Cho $\mathrm{H}$, et al. Usefulness of enhanced recovery after surgery protocol as compared with conventional perioperative care in gastric surgery. Gastric Cancer. 2012;15:34-41.

[Received November 22, 2019 : Accepted January 24, 2020] 